\title{
Advanced study of the thermoelectrically generated magnetic field in a nine-cell cavity during the superconducting phase transition
}

\author{
J.-M. Köszegi ${ }^{1}$ and J. Knobloch ${ }^{1,2}$ \\ ${ }^{1}$ Helmholtz-Zentrum Berlin, Albert-Einstein-Straße 15, 12489 Berlin, Germany \\ ${ }^{2}$ Universität Siegen, Walter-Flex-Str. 3, 57068 Siegen, Germany
}

(Received 19 December 2018; published 2 May 2019)

\begin{abstract}
Today's superconducting radio-frequency (srf) cavities approach quality factors up to $10^{11}$. With the decrease in overall dissipation the loss contributions previously considered minor gain in importance. One contribution is trapped magnetic flux. Superconducting rf cavities have to be cooled down to their cryogenic operating temperature. During the process, high temperature differences can occur in the system driving thermoelectric currents in the cavity wall and the surrounding liquid helium tank. The associated magnetic field can get trapped in the superconductor during its phase transition from the normal conducting into the superconducting (sc) state and significantly increase dissipation during later operation. The existence of this effect has been proven in general. The study presented here now adds experimental data and simulations on the amplitude and distribution of the trapped magnetic flux after the sc phase transition in the complete nine-cell cavity geometry. Furthermore, the dynamic behavior in the thermoelectrically generated magnetic field during the phase transition is evaluated.
\end{abstract}

DOI: 10.1103/PhysRevAccelBeams.22.052001

\section{INTRODUCTION}

For many modern accelerators, continuous wave superconducting radio-frequency (srf) cavities are a key component. Presently, significant research and development is concentrating on reducing the power dissipation in these cavities to reduce the often prohibitively large cryogenic load. During the process it was found that the surface resistance $R_{S}$ can be impacted by a number of different treatments. Various annealing techniques for high purity (high residual-resistivity ratio $\mathrm{RRR} \approx 300$ ) niobium with titanium or nitrogen were established in the past years [1,2]. They mainly influence the temperature and rf field dependence of the BCS surface resistance. In contrast, we expand here on studies to minimize the residual losses which remain at lowest temperatures.

It has been demonstrated that large temperature differences between the two cavity (niobium) and tank (titanium) joints drive thermoelectric currents during the cool down process in a horizontal cavity test assembly or cryomodule. The currents generate a magnetic field which can get trapped during the superconducting phase transition [3-5]. Any asymmetry in the temperature profile is important in determining the additional surface

Published by the American Physical Society under the terms of the Creative Commons Attribution 4.0 International license. Further distribution of this work must maintain attribution to the author(s) and the published article's title, journal citation, and DOI. resistance, which can be as high as $10 \mathrm{n} \Omega$, thus degrading significantly the quality factor of typical cavities [6]. While the correlation between increased temperature differences in the system and increased rf losses was intensely studied, a direct observation and assessment of the trapped magnetic flux in the cavity walls had not been presented so far. Thus the understanding of the effect was limited to indirect conclusions.

In this paper, we present a study that analyzes in detail the thermoelectrically generated magnetic field. We simulate numerically the field distribution in the whole cavitytank system for an asymmetric temperature distribution using a finite element simulation software package (COMSOL). The asymmetry causes the field to penetrate the rf surface which would be field free in the symmetric case. The calculated results are complemented by a direct measurement of the trapped magnetic flux inside the cavity with fluxgate magnetometers. The obtained data is combined with previous rf measurements in three passband modes to determine the overall distribution of the trapped magnetic flux due to thermocurrents.

In addition, the at times puzzling dynamic change in the magnetic field during the phase transition is both simulated and measured. Here, the goal is to understand the interaction of the thermocurrent with the growing superconducting area which exhibits no dc electrical resistivity and (almost) perfect diamagnetism. The simulations can explain characteristic features that are repeatedly observed during magnetic field measurements at cavities. Furthermore, we show that, based on the combination of 
simulations and measurements, the overall amount of thermocurrent in the system can be assessed with only one magnetic field probe if positioned correctly.

Besides the thermocurrents in the cavity-tank system, any connection between the $2 \mathrm{~K}$ system and room temperature holds the potential to generate additional thermocurrents which may add to the magnetic field at the cavities position. While the effect must be considered in the design of a module, it is not the subject of this paper.

\section{TRAPPED FLUX IN THE SC STATE}

The first section is dedicated to the calculation and thus understanding of the initial (steady state) magnetic field distribution prior to the superconducting (sc) phase transition. The simulations are supplemented by direct measurement of the magnetic field and, most importantly, the evaluation of the trapped magnetic flux in the sc material after the phase transition.

\section{A. Simulation}

Numerical simulations are a useful tool for understanding the distribution of the generated magnetic field in the whole setup. They allow us to attain a complete picture of our system and provide the information needed for the later experiment where we are limited to a small number of magnetic field probes.

\section{Setup}

Figure 1 presents the model which was used to compute the magnetic field in the system. It includes the niobium cavity, the tank which holds the liquid helium (LHe), and is fabricated from titanium. A magnetic shield around the helium vessel is included in the simulations. Couplers, tuner, and small features like brackets etc. are left out because they increase the number of mesh cells and thus the computation time without influencing the thermoelectric results.

The bellow that compensates length differences between tank and cavity caused by thermal contraction is omitted as well. However, it has to be accounted for in a different manner because it is fabricated from a thin titanium sheet $(\approx 0.3 \mathrm{~mm}$ thickness $)$ and its electrical resistance is comparable to the resistance of the tank. Hence, it increases the dc resistance of the overall system considerably and limits the thermocurrent as was discussed in Ref. [7]. In

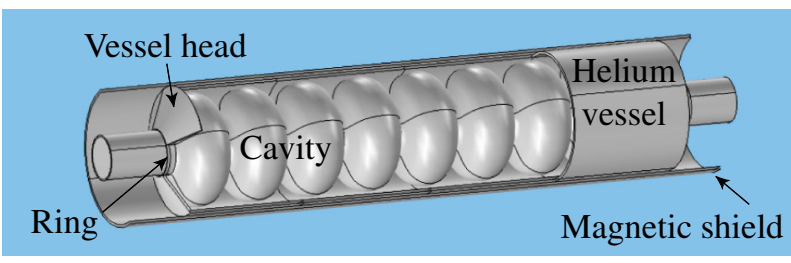

FIG. 1. COMSOL model of cavity, magnetic shield, and tank with ring for adjustment of electrical resistance. addition, the helium vessel heads are fabricated from niobium-titanium (NbTi), not titanium (Ti) as is assumed in the model. Literature data on the NbTi used in srf cavities is incomplete and therefore we refrained from implementing the material in the simulations. Nevertheless, the vessel heads increase the dc resistance of the system, just as the bellow, leading to a decrease in thermocurrent.

This increase in resistance is accounted for by implementing a small ring between niobium and titanium at one end of the cavity in the model. The ring is assigned titanium material properties with an exception to the electric conductivity, which was left scalable. The total resistance of the cavity-tank system is then tuned using this ring to match the experimental data in Ref. [8].

The material properties needed for the simulations are the heat capacity, the thermal conductivity, the electrical conductivity, and the thermopower. The temperature dependent values are taken from Refs. [9-11].

Besides the material properties, the overall thermocurrent in the cavity-tank system is determined by the temperatures of the two niobium-titanium joints. We set the temperatures of the joints to 10 and $80 \mathrm{~K}$ to model a cool down process with a high temperature difference.

The goal of the simulations is to understand the amount and distribution of the magnetic field that is generated by the thermocurrent. We are especially interested in the magnetic field at the rf surface which is the inner surface of the cavity. Only the magnetic flux that gets trapped at this location causes dissipation during later operation.

While the overall thermocurrent is mainly defined by the material properties and the temperatures of the two joints, previous studies have demonstrated that the magnetic field at the rf surface is strongly dependent on the temperature distribution [4,5]. Figure 2 depicts the distribution of the thermocurrent for two different temperature configurations. In the upper, symmetric case, the magnetic field is mainly located in between cavity and tank due to the mechanical

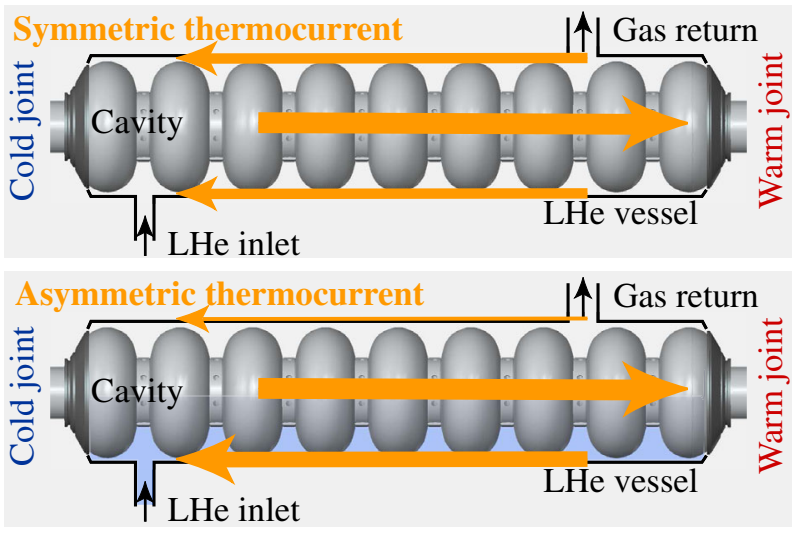

FIG. 2. Thermocurrent in the cavity-tank system when the two joints of $\mathrm{Nb}$ and $\mathrm{Ti}$ are at different temperatures. Top: symmetric current. Bottom: temperature gradient caused by the coolant is considered resulting in an asymmetric distribution. 


\section{Temperature boundaries}

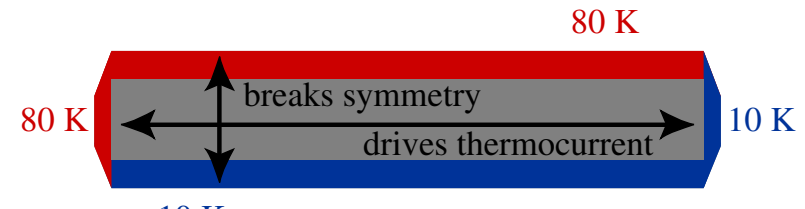

$10 \mathrm{~K}$

\section{Resulting magnetic field}
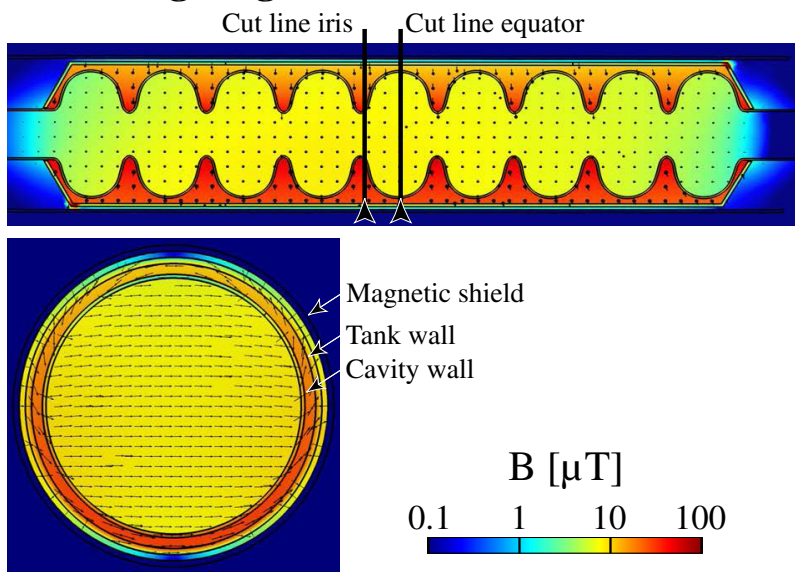

Magnetic shield

Tank wall

Cavity wall

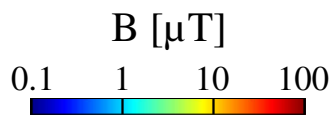

FIG. 3. Simulation of the magnetic field generated by a thermocurrent in an asymmetric temperature distribution. In the following graphs, cuts of the field are shown either along the cavity axis or along the cut lines indicated in the middle plot. In the bottom plot the field in the equator cross section is depicted.

symmetry of the coaxial cavity-tank setup. The magnetic field decreases inside the cavity wall towards the rf surface and drops to zero at the inner surface.

In the lower case in Fig. 2, a second temperature gradient in the system originates from the fact that the coolant ( $\mathrm{LHe}$ ) always collects at the bottom of the tank first. Thus a bottom to top gradient establishes in addition to the gradient along the cavity axis. It leads to a local variation of electrical resistivity and therefore the current distribution becomes asymmetric. A significant magnetic field appears at the rf surface.

Both temperature gradients were implemented as temperature boundary conditions in the simulation to obtain a realistic estimate of the magnetic field in the system right before the start of the sc phase transition.

\section{Results}

Figure 3 shows the implemented temperature boundary conditions on the tank together with the calculated magnetic field in the whole system. Due to the large range, a logarithmic scale was chosen.

We can already draw several conclusions from this initial computation. First, the figure shows that the asymmetry in temperature causes an asymmetry in the magnetic field between cavity and tank. For better understanding, two cut

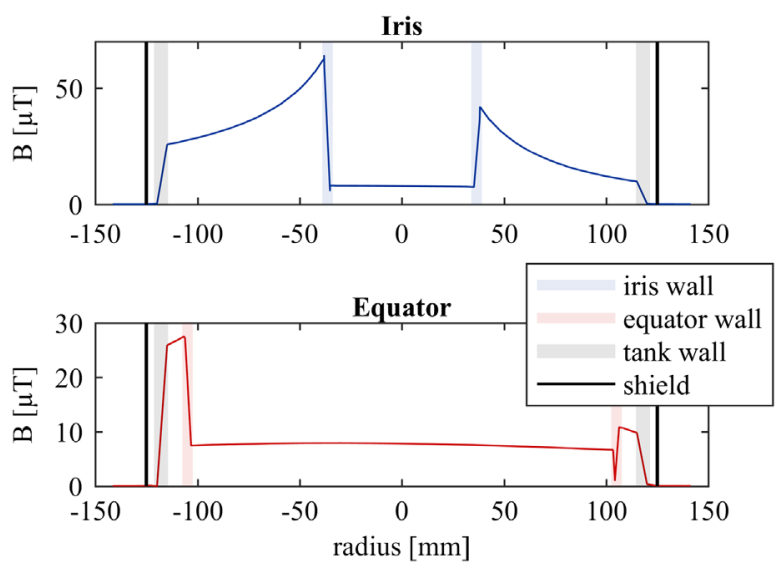

FIG. 4. Absolute magnetic field as a function of distance from cavity axis (radius). Values were exported along cut lines from bottom to top at the iris and the equator of cell 5 .

lines were drawn from bottom to top, one at the iris and one at the equator of cell 5 . The absolute magnetic field exported along the two cuts is plotted in Fig. 4.

It is shown that the magnetic field at the bottom (negative radius values) of the system is increased compared to the magnetic field at the top (positive radius values). The maximum values are reached at the outer surface of the cavity. Inside the cavity wall, the magnetic field decreases but it does not vanish at the inner surface. It passes into an almost homogeneous magnetic field in the cavity volume.

Figures 3 and 4 show nicely how the value of the magnetic field inside the cavity volume is almost constant which can be explained by the magnetic shield surrounding the whole system. The magnetic field decreases only slightly from bottom to top due to the asymmetric distribution between cavity and tank. Furthermore, it decreases towards the end cells which is caused by the temperature boundary conditions: The helium vessel heads are assigned symmetric boundaries hence the asymmetry in the temperature distribution and with it the magnetic field inside the cavity decrease towards the ends.

The magnetic field inside the cavity amounts to 5-8 $\mu \mathrm{T}$ and is therefore in the right magnitude to explain the observed changes in surface resistance in previous rf measurements.

Furthermore, the simulations reveal the orientation of the thermoelectrically generated magnetic field. The field lines inside the cavity are orthogonal to both the transverse and longitudinal temperature gradients. Based on this result, a dedicated measurement for the thermocurrent field can be set up.

\section{B. Measurement}

The direct experimental proof of thermoelectrically generated trapped magnetic flux has the major drawback that the field has to be measured inside the cavity and cavity operation is impossible. Nevertheless, we chose to perform a measurement of the trapped magnetic flux inside the 


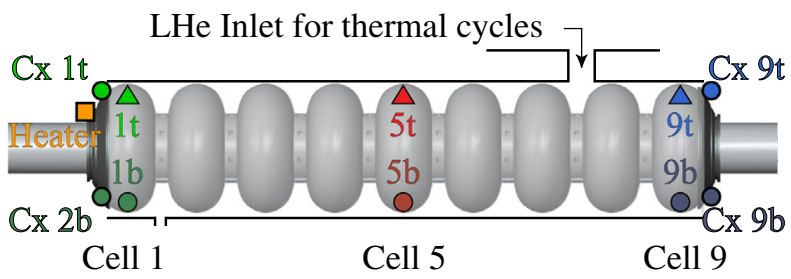

FIG. 5. Positions of temperature sensors $(\mathrm{Cx})$ and six fluxgate magnetometers (FG) in the direct measurement of trapped flux inside the cavity. Triangles indicate FGs in the top of the cells; circles indicate FGs in the bottom. The tank is filled with LHe via the two phase pipe.

cavity using six fluxgate magnetometers (FG). The cavity was provided by DESY. The acquired data was compared with previous rf measurements published in Ref. [4].

\section{Setup}

The setup of the experiment is depicted in Fig. 5. Four temperature sensors (Cernox sensors, $\mathrm{Cx}$ ) are mounted on the helium vessel heads at the same positions as in previous rf tests [4]. The sensors are used to measure the temperature difference between the two $\mathrm{Nb}$-Ti joints which drive the thermocurrent during the cool down process.

In addition, six FGs are positioned inside cells 1, 5, and 9 at the equators in perpendicular orientation with respect to the cavity axis as shown in the figure. For installation, a rod of rectangular cross section and equipped with blocks was inserted at the cavity axis. The fluxgates were mounted on the spring-loaded blocks. The springs were released and the fluxgates thereby installed at their respective positions. The mechanism fixed the angle of the fluxgate. Due to the cosine dependence of the field projection on the fluxgate angle, the setup is error tolerant to the extent of conceivable misalignments.

For better orientation in subsequent graphs, the sensors are color and marker coded. Each FG measures only in one spatial direction that is in the azimuthal direction in Fig. 5. Hence the setup can only detect a magnetic field arising from thermocurrents in an orientation predicted by the simulation (Fig. 3). The residual earth magnetic field in almost not detectable. Before cool down the FG probes measured values between $0.15 \mu \mathrm{T}$ and $7 \mathrm{nT}$. Furthermore, it must be noted that this configuration and hence also the data analysis differs significantly from a setup evaluating flux trapping outside a cavity.

Initially, the setup is cooled from room temperature to operation temperature (2 K). Subsequently, several thermal cycles are performed. During a thermal cycle the sc cavity is warmed up to temperatures around $20 \mathrm{~K}$ which exceeds the transition temperature of niobium $(9.2 \mathrm{~K})$. The tank is furthermore equipped with a heater to deliberately increase the temperature difference to $\Delta T \approx 70 \mathrm{~K}$ maximum. Afterwards, the cavity is cooled down again.
Finally, the trapped magnetic flux measured in the sc state is measured and correlated with $\Delta T$, the temperature difference between the two ends of the cavity tank system during the sc phase transition.

\section{Results}

Altogether, 10 thermal cycles are performed and during each cycle the average temperature difference $\Delta T$ is determined. It is calculated as an average from the four Cx values as $\Delta T=\left|\left(T_{\mathrm{Cx} 1}+T_{\mathrm{Cx} 2}\right) / 2-\left(T_{\mathrm{Cx} 3}+T_{\mathrm{Cx} 4}\right) / 2\right|$ when the first of the four sensors drops below the transition temperature. Figure 6 shows the level of the trapped magnetic flux as a function of $\Delta T$ where the trapped flux was measured after each thermal cycle once thermal equilibrium was reached.

The cell trapping the most magnetic flux is cell 9 which is also the cell closest to the LHe inlet, via the two phase pipe, and therefore the first cell to transition. Cells 5 and 1 show significantly reduced levels of trapped flux. In order to better understand the observation, Fig. 7 shows the signal of four FGs during the phase transition in one generic thermal cycle.

FG9b is the first FG to indicate a phase transition by a jump in magnetic field (Meissner effect). It is followed by FG9t only 2-3 sec later. FG9t is the sensor positioned closest to the two phase pipe. During all cycles with a $\Delta T$ larger than $20 \mathrm{~K}$, the sensors in cell 9 are the first to indicate a transition into the sc state and cell 9 traps the most magnetic flux. The maximum values of trapped flux in the test are $8.3 \mu \mathrm{T}$ (FG9b) and $6.6 \mu \mathrm{T}$ (FG9t) for a thermal cycle with $\Delta T \approx 70 \mathrm{~K}$. The simulation presented in Figs. 3 and 4 yielded magnetic field values of $7.9 \mu \mathrm{T}$ (FG9b position) and $6.7 \mu \mathrm{T}$ (FG9t position) which is a remarkable agreement.

In Fig. 7 we also see that cell 5 transitions roughly 2 min after cell 9. The cell exhibits a maximum level of trapped flux of $2.4 \mu \mathrm{T}$. Finally, cell 1 transitions almost 10 min after cell 9. The trapped flux is below $0.35 \mu \mathrm{T}$ after all cycles.
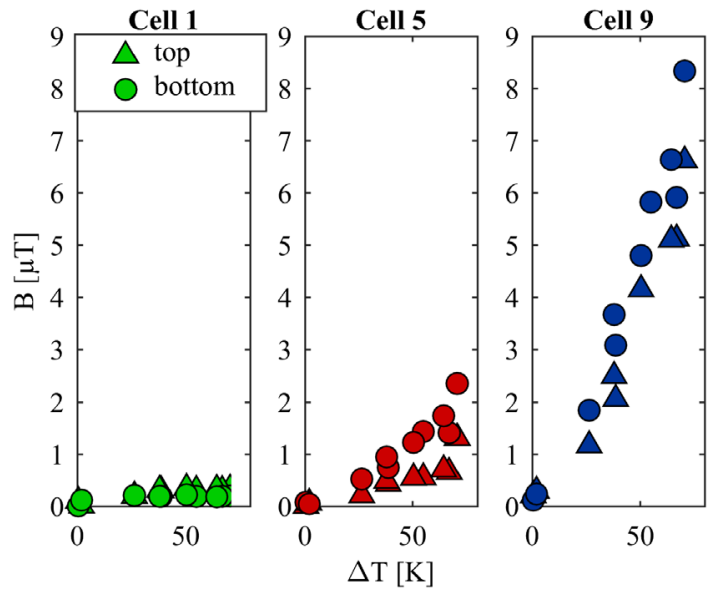

FIG. 6. Trapped magnetic flux measured at $2 \mathrm{~K}$ by six FGs after 10 thermal cycles. 


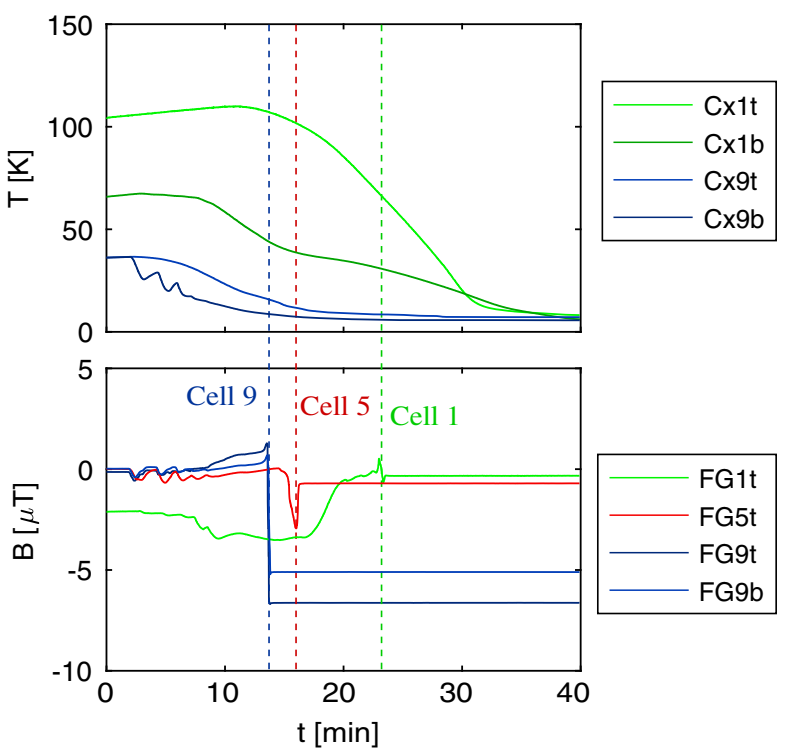

FIG. 7. Temperature and magnetic field during one generic cycle. The dashed lines indicate the phase transitions of the respective cells.

Furthermore, the figure shows that the temperature of the hot joint decreases rapidly during the time in which the sc phase expands from cell 9 to cell 1 until the whole cavity is superconducting. The thermopower $S$ of $\mathrm{Nb}$ exhibits its maximum at $\approx 72 \mathrm{~K}$ which corresponds to the average temperature of the hot point at the onset of the phase transition. Since $S$ and $\Delta T$ are both decreasing in that period, the thermocurrent in the system decreases as well. A reduced thermocurrent generates lower levels of magnetic field which explains the lower levels of trapped flux in cells 5 and 1 .

In addition to the distribution of trapped flux along the cavity, we see that the amount of trapped flux increased with $\Delta T$ for cells 5 and 9 , which is as expected. A higher temperature difference generates a higher thermocurrent and hence a higher magnetic field. The data shows that the trapped flux increases accordingly.

\section{Conclusion and comparison to $\mathrm{rf}$ measurements}

The data on the trapped magnetic flux in three cells can be combined with previously presented rf measurements [4] in order to estimate the distribution of the trapped magnetic flux in all nine cells.

The rf measurements had been performed in three passband modes: the accelerating $\pi$ mode, the $1 / 9 \pi$ mode, and the $8 / 9 \pi$ mode. All three modes have comparable resonance frequencies but different electric field distributions [12]. Hence the measured surface resistance is averaged over the nine cavity cells with different weightings. All cells have the same weight in the accelerating mode while the $1 / 9 \pi$ gives more weight to the center cells and the $8 / 9 \pi$ gives more weight to the end cells.
Trapped magnetic flux $[\mu \mathrm{T}]$
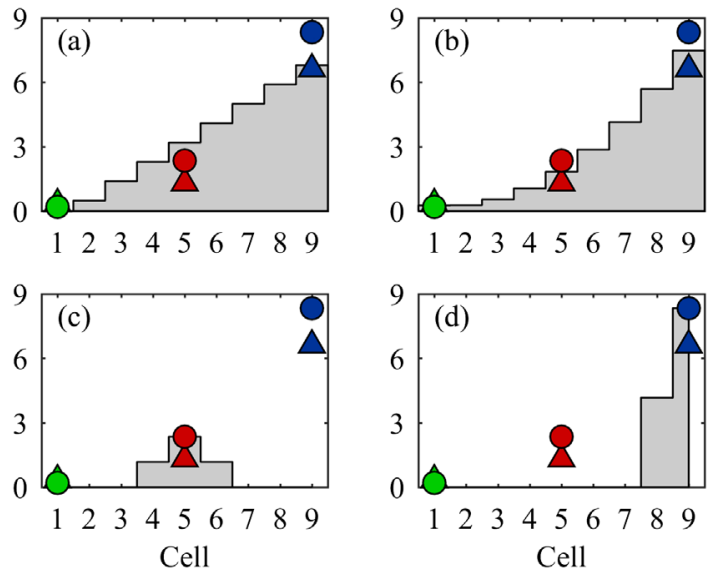

FIG. 8. Trapped magnetic flux data in three cells for one thermal cycle and several postulated interpolations for subsequent data analysis: (a) linear, (b) quadratic, (c) center cells, (d) end cells.

Based on the rf data, the previous study suggested that the thermoelectric contribution to rf dissipation is not a local effect but affects the cavity globally. The exact distribution was not determinable due to the mirror symmetry of the employed modes.

Now, we can go one step further and combine the rf data with the direct trapped flux measurement. The first step is to build possible trapped flux distributions in all nine cells based on the data in three cells in Fig. 6. Figure 8 shows a linear and a quadratic interpolation over the length of the cavity for one example thermal cycle. For comparison, two more interpolations are constructed which consider either only center or only end cells.

The interpolations from Fig. 8 are used to calculate the rf dissipation that would result in the three passband modes. Figure 9 reproduces the rf data from the study in Ref. [4] for the then performed 11 thermal cycles. The solid lines indicate linear fits to the rf data. The $1 / 9 \pi$ and $8 / 9 \pi$ have an offset which is caused by the shielded earth magnetic field in the cryostat. It is largest in the end cells due to the cutouts in the shield for the couplers etc. while the center cells are shielded best. The offset does not show up in the direct measurement of trapped flux in Fig. 8 due to the orientation of the FGs.

In addition to the rf data, Fig. 9 displays dashed lines that are calculated based on the interpolations in Fig. 8. The four investigated profiles (a)-(d) also yield linear increases of the $R_{\text {res }}$ average in the $1 / 9 \pi$ and $8 / 9 \pi$ mode versus the $\pi$ mode average. Table I gives the slopes of all four graphs and compares them to the slopes obtained from the rf data.

The combination of direct measurement of trapped flux and $\mathrm{rf}$ data allows us to assess the distribution of the thermoelectrically generated trapped magnetic flux over the whole cavity. The data clearly excludes distributions which 


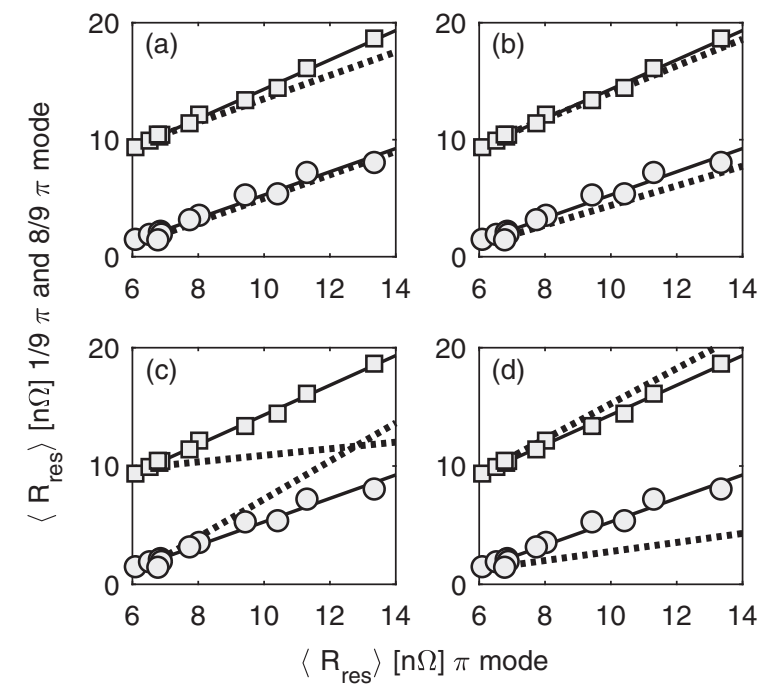

FIG. 9. Average $R_{\text {res }}$ in $1 / 9 \pi$ (circles) and $8 / 9 \pi$ (squares) mode as functions of accelerating $\pi$ mode $R_{\text {res }}$ average. Solid lines indicate linear fits to the rf data. Dashed lines show $R_{\text {res }}$ calculated from the trapped flux data in Fig. 8 with (a) linear, (b) quadratic, (c) center cell, and (d) end cell profiles.

only consider trapped flux localized in a few cells which is as expected and demonstrates the functionality of the method. A linear profile, however, reproduces the rf data of the $1 / 9 \pi$ mode and fits with the $8 / 9 \pi$ mode data in the double error margin. The $8 / 9 \pi$ mode data is better described by a quadratic profile which, in turn, deviates for the $1 / 9 \pi$ mode.

We conclude that the actual distribution of the trapped magnetic flux due to thermocurrents is in between linear and quadratic and, in any case, degrades the cavity surface resistance globally. Since both profiles yield reasonable results, we can even calculate the absolute amount of surface resistance that the directly measured trapped flux would cause. Table II displays the additional $R_{\text {res }}$ caused by the maximum trapped flux (for $\Delta T \approx 70 \mathrm{~K}$ ) and compares

TABLE I. Slopes calculated for $1 / 9 \pi$ and $8 / 9 \pi$ mode versus $\pi$ mode average $R_{\text {res }}$ (dashed lines in Fig. 9) together with the measured slope (solid line in Fig. 9).

\begin{tabular}{lccccc}
\hline \hline & rf data & (a) & (b) & (c) & (c) \\
\hline $1 / 9 \pi$ mode & $1.0 \pm 0.1$ & 1 & 0.8 & 1.6 & 0.4 \\
$8 / 9 \pi$ mode & $1.2 \pm 0.1$ & 1 & 1.1 & 0.3 & 1.5 \\
\hline \hline
\end{tabular}

TABLE II. Absolute values of $R_{\text {res }}$ increase measured in the rf test and calculated based on an (a) linear or (b) quadratic trapped flux profile (all with $\Delta T \approx 70 \mathrm{~K}$ during cooldown).

\begin{tabular}{lccc}
\hline \hline & rf data & (a) & (b) \\
\hline$\pi$ mode & $7.2 \mathrm{n} \Omega$ & $3.2 \mu \mathrm{T} \hat{=} 11.2 \mathrm{n} \Omega$ & $2.7 \mu \mathrm{T} \hat{=} 9.5 \mathrm{n} \Omega$ \\
$1 / 9 \pi$ mode & $9.2 \mathrm{n} \Omega$ & $3.2 \mu \mathrm{T} \hat{=} 11.2 \mathrm{n} \Omega$ & $2.3 \mu \mathrm{T} \hat{=} 8.1 \mathrm{n} \Omega$ \\
$8 / 9 \pi$ mode & $8.7 \mathrm{n} \Omega$ & $3.2 \mu \mathrm{T} \hat{=} 11.2 \mathrm{n} \Omega$ & $3.0 \mu \mathrm{T} \hat{=} 10.5 \mathrm{n} \Omega$ \\
\hline \hline
\end{tabular}

it to the actually measured increase in $R_{\text {res }}$ in the previous study [4]. The approximation of $3.5 \mathrm{n} \Omega / \mu \mathrm{T}$ was used based on Ref. [6].

The data shows remarkable agreement given the $10 \%$ error bar on the rf data and the limitations of the direct measurement (only six FGs in one spatial direction). Remaining differences might be attributed to the additional asymmetry introduced by the chimney of the helium tank, which was omitted in the simulation.

\section{SC PHASE TRANSITION}

The first part of this paper investigated the level of trapped magnetic flux in the sc state after the phase transition. The data was compared to a simulation which assumed an asymmetry in the temperature distribution over the whole length of the cavity (Fig. 3). The approximation yielded results consistent with the maximum level of the trapped magnetic flux observed in the measurement. However, the actual asymmetry in the system is largest once the first sc area establishes. Therefore, the dynamics of the magnetic field at the transition time and location are much more complicated which manifests in the experiments in the form of e.g. overshoots in the measured magnetic field.

In order to clarify open questions concerning the actual phase transition, we set up a second set of simulations investigating the asymmetry caused by the appearance of the first sc spot which subsequently grows. The results of the calculations are then compared to previous data which we presented in Ref. [13].

\section{A. Simulation}

In the simulations, the superconducting material is implemented in the form of niobium with modified material properties. The electrical conductivity is increased by a factor of $10^{3}$ and $\mu_{r}$ is set to $10^{-6}$ to simulate (almost) vanishing $\mathrm{dc}$ resistance and perfect diamagnetism. The thermal properties are not changed.

The goal of the simulations is to investigate the influence of the localized appearance of superconductivity independently of other sources of asymmetry. Hence, the setup is designed based on a symmetric temperature distribution, which provides the same driving force for the thermocurrent as in the previous simulations but without embedded asymmetry.

A round area at the bottom of the equator of cell 5 is defined and set to the modified niobium properties to simulate the superconducting spot without manipulating the temperature distribution. Thereby, the superconducting area is not below the transition temperature, which does not matter because the properties are artificially set superconducting.

The setup of the simulation is shown in Fig. 10. It allows an estimate of the field distribution after the onset of phase transition without the necessity of implementing a 


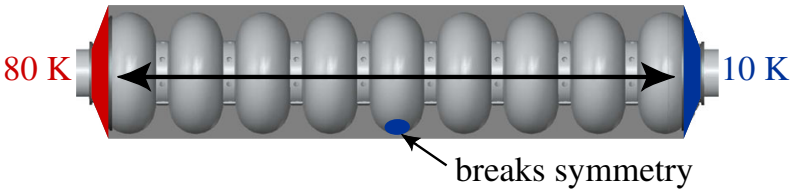

FIG. 10. Setup for the calculation of the magnetic field distribution caused by a thermocurrent considering a growing sc spot. The spot was positioned at the center cell to minimize the impact of the boundary conditions.

complicated temperature distribution. The simulation was repeated for several postulated sizes of the superconducting region. Hence the sequence can be viewed as a superconducting region growing in time.

Figure 11 shows the results of the simulations. The numbers give the diameter of the spot and the black dot in the first plot gives the "FG position" at which the magnetic field values are compared.

In the symmetric case before the sc transition ( $0 \mathrm{~mm}$ spot diameter), the field value is $17.9 \mu \mathrm{T}$. For the smallest sc spot simulated $(12 \mathrm{~mm})$, the field increases to $20.3 \mu \mathrm{T}$. At the edge of the superconducting area, the field even reaches a value of $28.7 \mu \mathrm{T}$. With increasing spot size, the magnetic field at the FG position decreases towards the value of the symmetric case. The field at the edge, however, increases due to the implemented Meissner effect. The data is displayed in Fig. 12.

\section{B. Measurement}

The data presented in Fig. 12 can be compared to previous direct measurements of the magnetic field in Ref. [13]. Figure 13 displays the sensor positions in this study. The temperature was measured and $\Delta T$ calculated as

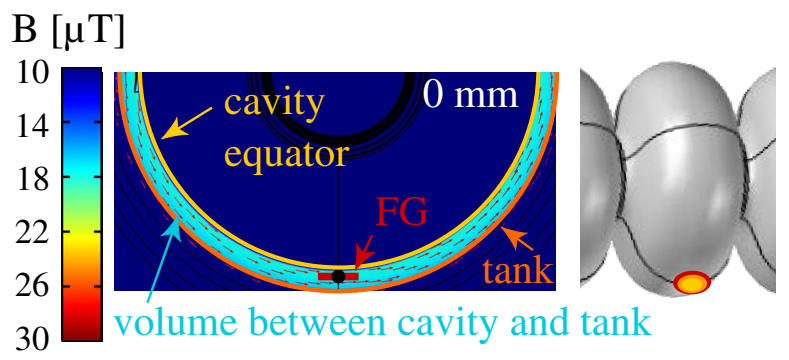

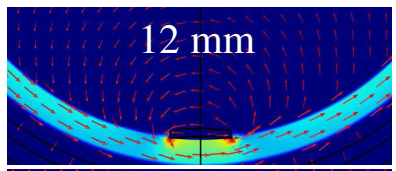
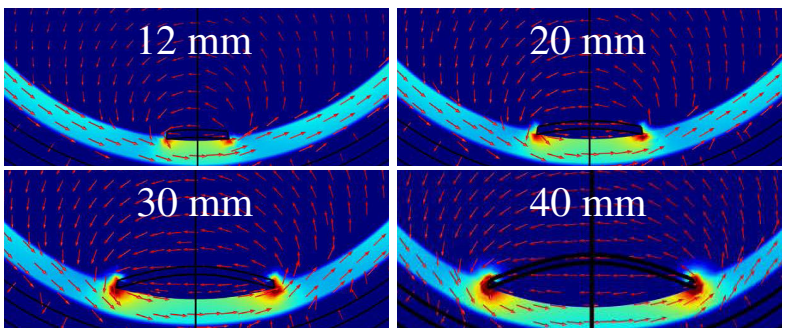

FIG. 11. Magnetic field distribution around a growing sc spot. Black dot on top of FG in first graph indicates "FG position" used for evaluation in following plots. White numbers indicate the diameter of the sc spot in the respective simulation.

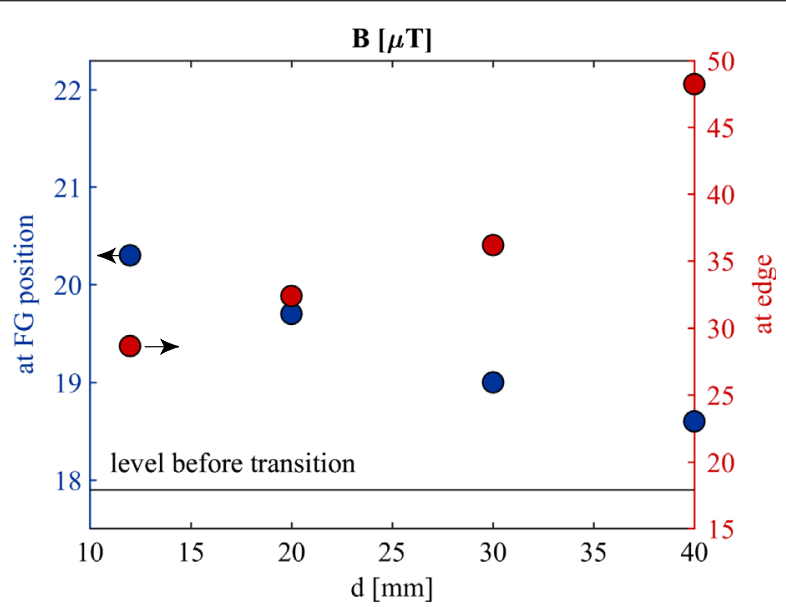

Simulation:

at the FG position

at the edge of the spot

FIG. 12. Magnetic field of a growing sc spot at the FG position and the maximum field at the edge of the sc spot.

explained in Sec. II B. The FG, however, was positioned inside the tank and not inside the cavity. The benefit of measuring in the tank is that the instrumentation does not interfere with any rf tests performed on the cavity. The drawback is that the measurement mainly gives information on the thermoelectriaclly generated magnetic field in the complete system but not specifically at the rf surface.

Figure 14 shows the signal of the magnetic field probe inside the tank during two representative thermal cycles. $\Delta T$ was calculated when the first temperature sensor dropped below the transition temperature.

In cycle $\mathrm{A}$ there was only a small temperature difference in the system $(\Delta T=2.5 \mathrm{~K})$. Due to the rather homogeneous transition, $\Delta T$ even inverted the sign during the cool down. Accordingly the measured magnetic field values are low. In cycle B, however, a large temperature difference was present $(\Delta T=68 \mathrm{~K})$ leading to a significantly larger magnetic field.

For all cycles with $\Delta T$ values larger than $20 \mathrm{~K}$, an almost constant magnetic field value was reached in the tank prior to the phase transition. Once the transition started, the temperature difference monotonically decreased but the magnetic field exhibited a peak $B_{\text {peak }}$ before it also

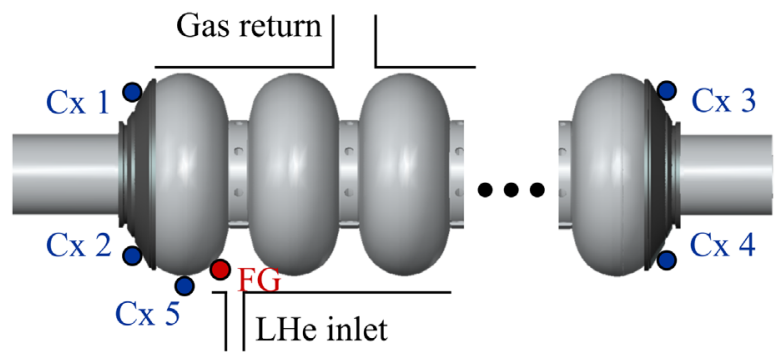

FIG. 13. Temperature sensor (Cx) and fluxgate magnetometer (FG) positions in the experiments in Ref. [13]. 

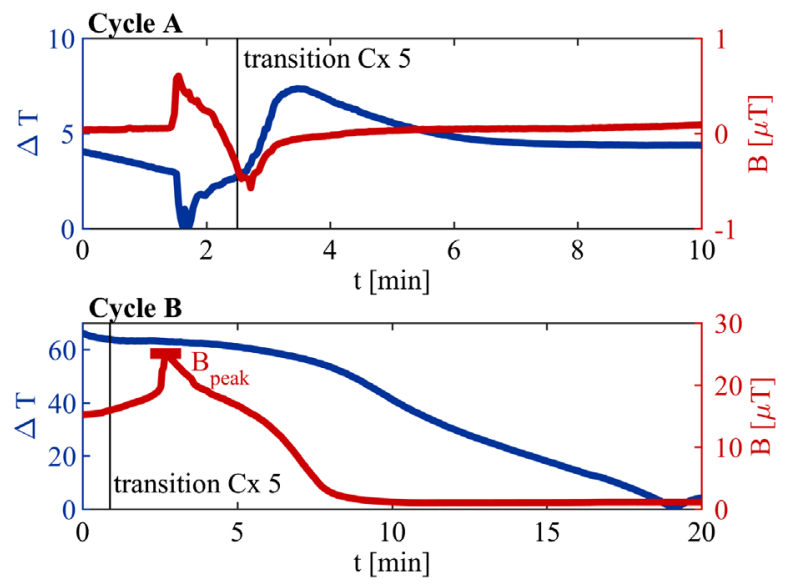

FIG. 14. Temperature difference along the system and magnetic field signal during two thermal cycles. Black vertical lines indicate the time when $\mathrm{Cx} 5$ close to the FG dropped below the transition temperature.

decreased. The peak is shown in Fig. 14 and also in Fig. 15 together with the peaks that occurred during two similar cycles. The feature was very reproducible for any thermal cycle with a significant $\Delta T$ along the cavity-tank system. Furthermore, its amplitude was directly correlated to the rf dissipation as was demonstrated in Ref. [13]. We therefore conclude that the peak value is a measure of the amount of thermocurrent in the complete system.

The origin of the peak can be understood based on the presented simulations. The left panel in Fig. 15 shows the FG data of cycle B together with the magnetic field values that were extracted from the simulations of the growing sc spot. The simulation does not include time information. Hence the simulated data points can only be included in Fig. 15 as estimations. The figure was compiled as follows.
The spreads of both ordinates were set equivalent but the limits were shifted in a way that the measured value before the peak coincided with the simulated value of the symmetric simulation. The experimental data shows how the magnetic field present before transition gets enhanced once a sc spot establishes. The $30 \mathrm{~s}$ around the peak are marked yellow. This period corresponds to the experimental situation when the phase front is passing by the FG and accordingly the measured field exhibits a maximum.

As an approximation from the simulation, the maximum value computed at the edge for the smallest area size $(d=12 \mathrm{~mm})$ is added to Fig. 15 as an estimation of the peak height (red circle).

As the sc area grows, the magnetic field measured at the FG location decreases. To link the experimental data with the simulations, the calculated values of $d=20,30,40 \mathrm{~mm}$ are plotted equidistantly into the figure to describe the reduction in measured field when the phase front advances and hence its distance to the FG increases. The simulations and the data agree qualitatively very well, though it must be repeated that the simulated points have no direct relation to the time axis.

While Fig. 12 shows how the simulated magnetic field at the FG location is approaching the value before transition as the spot is growing, the measured field does not drop back to the value obtained before transition after the phase front passed but keeps decreasing. The decay is caused by the reduction of the joint temperatures during the proceeding cool down process. The reduced temperature difference and the lower $S$ values lead to a decrease in the overall amount of thermocurrent until it vanishes completely and only the magnetic flux which got trapped remains.

Based on the presented results of the simulations and the direct measurement we can understand the correlation of $\mathrm{rf}$

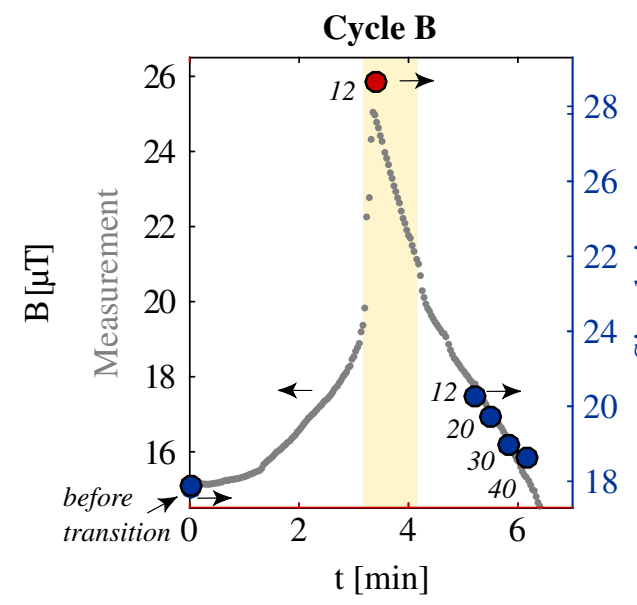

Measurement:
Simulation:
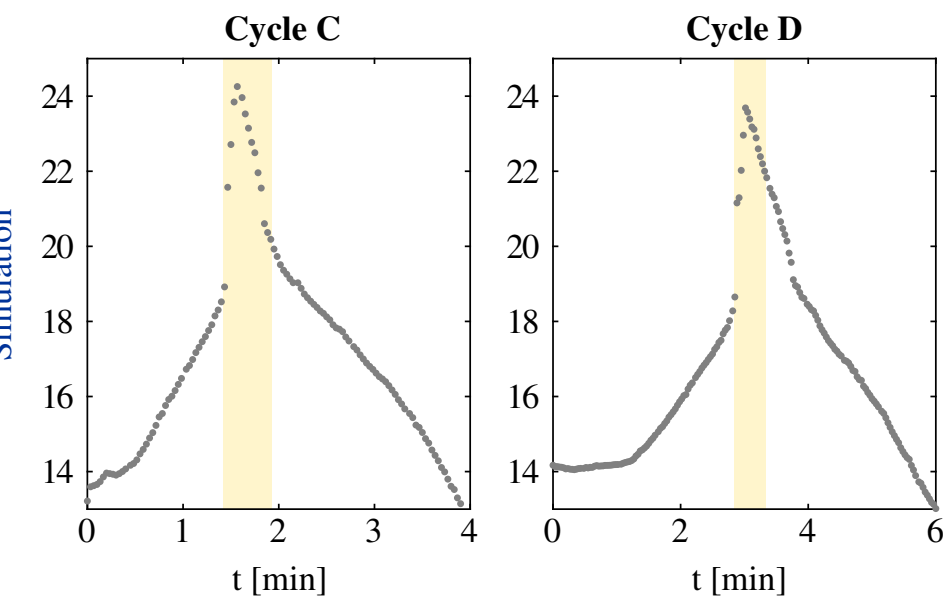

Cycle D

FIG. 15. Magnetic field peaks observed in several measurements (gray dots) together with simulated magnetic field values. Blue circles indicate field at the FG position for different sc spot sizes, diameters [mm] given as italic black numbers. Red circle inidcates field value at the edge of the smallest simulated spot size. 
dissipation and $B_{\text {peak }}$ identified in Ref. [13]. The change in $B_{\text {peak }}$ between different cycles, if measured at the same position and for similar relative temperature distributions, corresponds to the change in the overall amount of thermocurrent in the system. The more thermocurrent is flowing, the higher is the resulting peak in magnetic field during transition and vice versa.

While the general correlation between the peak in magnetic field and the thermoelectric current could be established, the absolute numbers of the measurement compared to the simulations need to be discussed further. The sensitive core of the fluxgate has a length comparable to the spot size in the simulations. Furthermore, the surface of the cavity is curved. We chose to compare experimental data from the fluxgate to specific locations in the simulations to illustrate a trend. Since the fluxgate will detect changes in the center of the spot as well as at its edge as long as the spot is of approximately the same size as the fluxgate, the values can only be understood as an indication of this trend.

\section{SUMMARY}

We presented a study combining numerical simulations and experimental data to assess the distribution, amplitude, and orientation of trapped flux caused by thermoelectrically generated magnetic field in a multicell cavity.

In combination with previously published rf data it was concluded that the actual distribution of the trapped magnetic flux due to thermocurrents is in between a linear and quadratic increase from almost zero at one end of the system to the maximum value at the opposite end. In any case, the cavity surface resistance is degraded globally.

The analysis allowed a detailed understanding of the effect especially during the sc phase transition. Based on the present results, only limited diagnostics in the LHe tank of any cavity is needed to correctly assess the overall thermocurrent in the system and with that to avoid degradation of the surface resistance.

The study did not address thermocurrents which occur outside of the cavity-tank system and originate from junctions, e.g. leads, connecting the $2 \mathrm{~K}$ system with room temperature. Depending on the amplitude of these currents the effect must also be considered in the design of a cryo module.

\section{ACKNOWLEDGMENTS}

We thank Axel Matheisen and his colleagues from DESY for providing a cavity which we could instrument from the inside.
[1] P. Dhakal, G. Ciovati, G. R. Myneni, K. E. Gray, N. Groll, P. Maheshwari, D. M. McRae, R. Pike, T. Proslier, F. Stevie, R. P. Walsh, Q. Yang, and J. Zasadzinzki, Effect of high temperature heat treatments on the quality factor of a large-grain superconducting radio-frequency niobium cavity, Phys. Rev. ST Accel. Beams 16, 042001 (2013).

[2] A. Grassellino, A. Romanenko, D. Sergatskov, O. Melnychuk, Y. Trenikhina, A. Crawford, A. Rowe, M. Wong, T. Khabiboulline, and F Barkov, Nitrogen and argon doping of niobium for superconducting radio frequency cavities: A pathway to highly efficient accelerating structures, Supercond. Sci. Technol. 26, 102001 (2013).

[3] J.-M. Vogt, O. Kugeler, and J. Knobloch, Impact of cooldown conditions at tc on the superconducting rf cavity quality factor, Phys. Rev. ST Accel. Beams 16, 102002 (2013).

[4] J.-M. Vogt, O. Kugeler, and J. Knobloch, High-Q operation of superconducting rf cavities: Potential impact of thermocurrents on the rf surface resistance, Phys. Rev. ST Accel. Beams 18, 042001 (2015).

[5] R. Eichhorn, C. Daly, F. Furuta, A. Ganshyn, M. Ge, D. Gonnella, D. Hall, V. Ho, G. H. Hoffstaetter, M. Liepe, J. May-Mann, T. O'Connell, S. Posen, P. Quigley, J. Sears, and V. Veshcherevich, Thermocurrents and their role in high Q cavity performance, Phys. Rev. Accel. Beams 19, 012001 (2016).

[6] B. Aune et al., Superconducting TESLA cavities, Phys. Rev. ST Accel. Beams 3, 092001 (2000).

[7] A. C. Crawford, A study of thermocurrent induced magnetic fields in ILC cavities, arXiv:1403.7996v1.

[8] J. Vogt (TESLA Technology Collaboration), Fermilab studies of quality factor changes of a $\mathrm{N}$ doped cavity from vertical to dressed horizontal test, TTC meeting, (KEK, 2014), https://tesla-new.desy.de/meetings/collaboration_ meetings_and_ttc_workshos/.

[9] J. M. Köszegi, Surface resistance minimization in srf cavities by reduction of thermocurrents and trapped flux, Ph.D. thesis, Universität Siegen, 2017.

[10] J. E. Jensen, R. B. Stewart, W. A. Tuttle, H. Brechna, and A. G. Prodell, Selected Cryogenic Data Notebook (Brookhaven National Laboratory, New York, 1980), Vol. 2.

[11] M. Merio and T. Peterson, Material properties for engineering analysis of SRF cavities, Fermilab Specification: 5500.000-ES-371110, Rev. A, 2011.

[12] R Wanzenberg, Monopole, dipole and quadrupole passbands of the TESLA 9-cell cavity, Report No. DESYTESLA-2001-33, 2001.

[13] O. Kugeler, J. Knobloch, J. M. Köszegi, A. Grassellino, O. Melnychuk, A. Romanenko, and D. Sergatskov, Horizontal testing and thermal cycling of an N-doped TESLA type cavity, Proceedings of the 17th International Conference on RF Superconductivity (JACoW, Geneva, Switzerland, 2015), http://accelconf.web.cern.ch/AccelConf/SRF2015/ papers/mopb019.pdf. 